\title{
Enhancement of Phase Contrast at Low Spatial Frequency Using Phase Plate STEM
}

Misaki Tsubouchi and Hiroki Minoda

Tokyo University of Agriculture and Technology, Koganei, Tokyo, Japan

In phase plate Scanning Transmission Electron Microscopy (P-STEM), phase plate (PP) was set in the pre-sample position and it can modify phase distribution of the incident electron wave. Thin film PPs with an appropriate design can provided a desired phase distribution to the incident wave and can control a phase contrast transfer function (PCTF) of the microscope. We used Zernike PP (ZPP) which has a hole at the center for P-STEM imaging. The thickness of the PP was chosen to provide a half pi phase shift for $200 \mathrm{kV}$ electron wave and the sine type PCTF was modified to the cosine type. One of the important targets to use PP is the structure with relatively low spatial frequency (lower than about $1 \mathrm{~nm}^{-1}$ ) such as bio materials. That's because the sine type PCTF of the conventional STEM is almost zero at low spatial frequency and image contrast of such samples can't be obtained. Our previous STEM study shows the validity of the ZPP to visualize nm scale materials [1,2].

The incident electron disc on the detector plane can be divided into two zones. One is inside of the hole image of the PP (A1 zone) and the other is the outside of the hole image of the PP (A2 zone). The optical condition of the electron waves land in the A1 zone and A2 zone are different. Since we have to use the unscattered electron wave without phase shift by the ZPP and the scattered waves with phase shift to obtain higher contrast image in P-STEM, the electron waves land onto the zone A1 have to be chosen. In the A1 zone the scattered waves are negatively interfere with reference or unscattered wave and sample images of the weak phase objects become darker than background intensity. On the other hand the scattered waves are positively interfere with the reference wave and sample images of the weak phase objects become darker than the background. The signals detected in the A2 zone provide the opposite contrast for those detected in the A1 zone.

One of the advantages to use two dimensional detector is to process the data after acquisition. And we can process the data according to the optical condition. By detecting the electron intensities landed in the A1 and the A2 zones separately and processing these to cancel the background intensity, we could dramatically improve image contrast. Figure 1 compares the images of multi wall carbon nanotubes (MWCNT) obtained using two dimensional detector. Fig. 1(a) was obtained by processing the signals detected by the A1 zone. The optical condition of this image is the same as that of conventional P-STEM which is obtained using a bright field detector with detector aperture. Fig. 1(b) was obtained by processing the signals detected by A1 and A2 zones. The image contrast of Fig. 1(b) is much higher than that of Fig. 1(a) and the superiority of the two dimensional detector is evident. In the presentation the details of the image processing and the investigation of further image improvements will be introduced. 

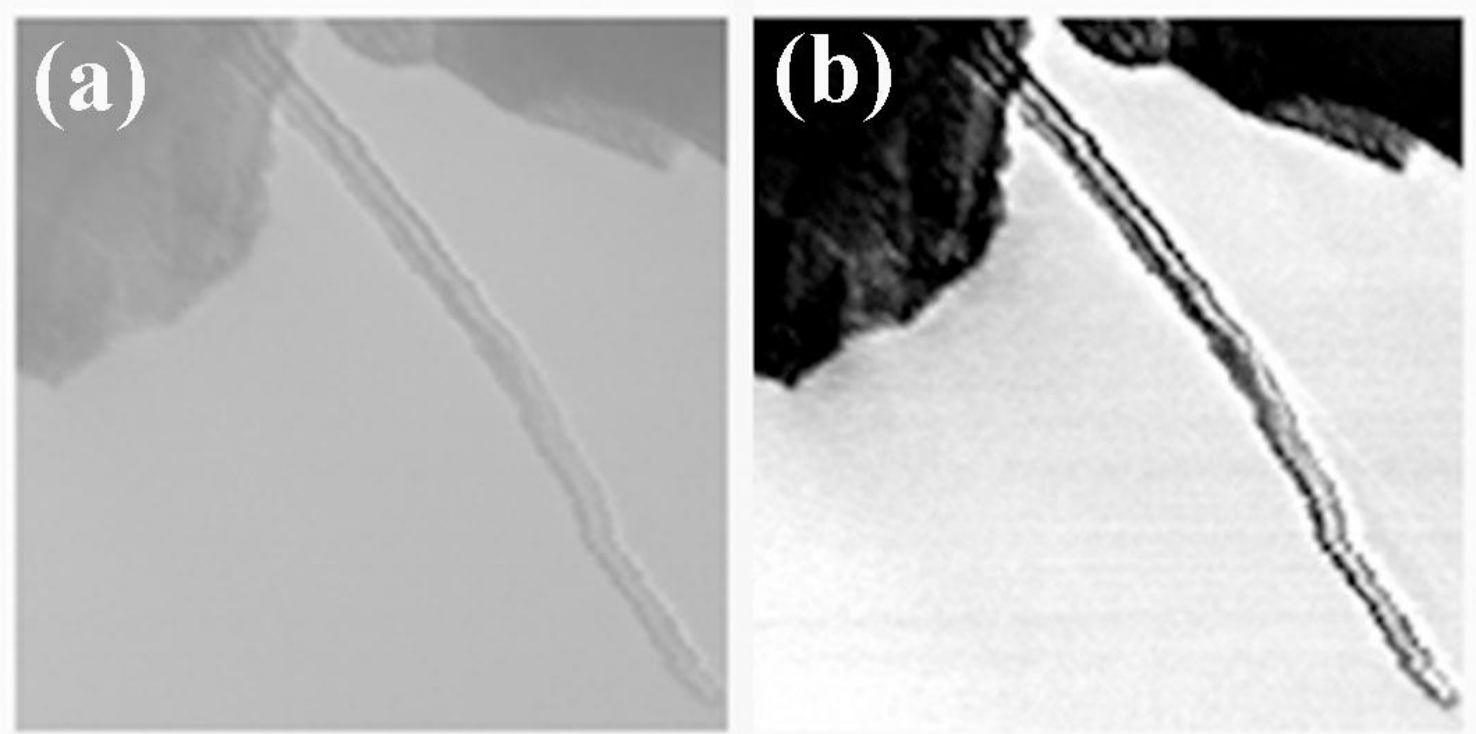

Figure 1. (a) P-STEM image of the MWCNT obtained by processing the signals detected by the A1 zone. (b) P-STEM image obtained by processing the signals detected by A1 and A2 zones. The optical condition of (a) is the same as that of the conventional P-STEM. Image contrast of MWCNT in (b) is much higher than that in (a).

References

[1] H. Minoda, T. Tamai, H. Iijima, F. Hosokawa and Y. Kondo, Microscopy 64 (2015) 181.

[2] H. Minoda, T. Tamai, Y. Ohmori and H. Iijima, Ultramicroscopy 182 (2017) 163. 